\title{
Nonlinear waves in disordered chains: probing the limits of chaos and spreading
}

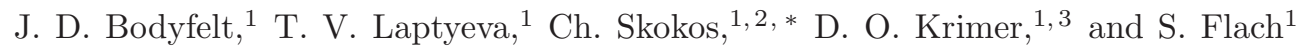 \\ ${ }^{1}$ Max Planck Institute for the Physics of Complex Systems, Nöthnitzer Str. 38, D-01187 Dresden, Germany \\ ${ }^{2}$ Center for Research and Applications of Nonlinear Systems, University of Patras, GR-26500, Patras, Greece \\ ${ }^{3}$ Theoretische Physik, Universität Tübingen, D-72076 Tübingen, Germany
}

(Dated: July 7, 2018)

\begin{abstract}
We probe the limits of nonlinear wave spreading in disordered chains which are known to localize linear waves. We particularly extend recent studies on the regimes of strong and weak chaos during subdiffusive spreading of wave packets [EPL 91, 30001 (2010)] and consider strong disorder, which favors Anderson localization. We probe the limit of infinite disorder strength and study FröhlichSpencer-Wayne models. We find that the assumption of chaotic wave packet dynamics and its impact on spreading is in accord with all studied cases. Spreading appears to be asymptotic, without any observable slowing down. We also consider chains with spatially inhomogeneous nonlinearity which give further support to our findings and conclusions.

PACS numbers: 05.45.-a, 05.60.Cd, 63.20.Pw
\end{abstract}

\section{INTRODUCTION}

Anderson localization [1] was discovered 50 years ago in disordered crystals as an accumulation of single particle electronic wavefunctions and can be interpreted as an interference effect between multiple scatterings of the electron by random defects of the potential. As a consequence eigenstates are no longer spatially extended, but exponentially localized. Anderson localization is a universal phenomenon of wave physics, unrestricted to quantum mechanics. Experimental observations were made in noninteracting Bose-Einstein condensates (BEC) expanding in random optical potentials [2, 3], light propagation in spatially random nonlinear optical media [4, 5], and in microwave cavities filled with randomly distributed scatterers [6]. Anderson localization is a linear wave effect, i.e. it is well-established for wave equations which are linear in the wave amplitude. However, in many cases one is confronted with a nonlinear response of the wave-carrying medium; for instance, large light intensities induce a nonlinear response of the optical medium. Electron-electron and electron-phonon interactions also result in substantial deviations from Anderson localization in solids. In experiments of BoseEinstein condensates the interatomic interactions are always present, although they can be diminished by either decreasing atomic densities or by exploiting magnetically tunable Feshbach resonances.

From a mathematical perspective, a linear wave equation is integrable, with each normal mode evolving independently in time. A localized wave packet in the presence of Anderson localization will therefore stay localized as time evolves. Nonlinearity will usually destroy the integrability of a system and induce mode-mode interactions. It was observed numerically that wave packets in such nonlinear disordered wave equations delocalize in

*Electronic address: hskokos@pks.mpg.de Corresponding author. time without respecting Anderson localization limits 7 11]. Thus, there are several intriguing questions which have attracted much attention during the last few years: (i) will Anderson localization be destroyed by arbitrary small strength of nonlinearity or is there a threshold below which the localization is restored? (ii) will wave packet spreading, if observed, last forever or will it stop at certain (though probably very large) time? (iii) is the shape of the initial wave packet crucial for the details of spreading? We will mainly address question (ii) here.

Johansson et al. 12] conjectured that spreading must eventually stop and dynamics will become close to regular, assuming that in these limits the KolmogorovArnold-Moser (KAM) theorem is applicable, i.e. that for small wave density regular nonergodic phase space structures predominate and the dynamics develops along KAM tori. Other attempts consist in a numerical scaling analysis, in order to predict and extend results beyond computational ability [13]. Analytical studies perform perturbation theory to higher order by treating the strength of nonlinearity as a small parameter 14], conflicting with the explosive growth of secular terms in higher orders of perturbation theory. This theory states that for the disordered discrete nonlinear Schrödinger model with nonlinearity strength exceeding a finite threshold, any initial localized wave packet cannot fully spread to zero amplitudes at infinite time. In this case, a part of the excitation is selftrapped as a result of nonlinearity induced frequency shifts, which tune a localized excitation out of resonance with its surrounding non-excited linear modes. However, even in the case of strong nonlinearity, subdiffusion of the nonselftrapped part is observed [9]. When strong nonlinearities are avoided numerical studies showed a rather universal asymptotic subdiffusive spreading of initial single site excitations [8, 9, 15], which is characterized by a growth of the second moment of the wave packet as $t^{\alpha}$, with $\alpha \leq 1$ [9, 15]. The selftrapping theorem [16] holds irrespective of the strength of disorder, therefore it is reflecting the properties of a strongly nonlinear lattice wave 
equation, rather than peculiarities of waves propagating in disordered media. Also the selftrapping theorem is crucially depending on the presence of at least two integrals of motion, and fails for most nonlinear wave equations with only one integral of motion.

In [9] the observed wave packet spreading was assumed to be due to an incoherent excitation of the wave packet exterior, induced by the chaotic dynamics of the wave packet interior. The number of resonant modes in the packet was estimated by considering from quadruplet and triplet mode-mode interactions [17]. A generalization to higher dimensions $D$ and different nonlinearity powers were performed. This led to a quantitative prediction for the subdiffusive wave packet spreading characteristics $\alpha$ [9]. Its validity was confirmed numerically in $9,15,18$. Recently, it has been predicted theoretically [19, 20] and verified numerically [19] that a potentially long-lasting strong chaos regime induces faster (though still subdiffusive) spreading, which is followed by the asymptotic and slower weak chaos subdiffusive spreading. Notably, published numerical data did not report on a further slowing down of spreading, when starting from the weak chaos regime.

In this paper we present results of extensive numerical studies of wave packet spreading in various models of disordered nonlinear one-dimensional lattices. In particular, we consider different initial excitations and scan the parameter space of disorder strength and nonlinearity over a wide region. The main aim is to test the applicability of previously derived spreading laws, and to search for indications of a continuation of the weak chaos spreading, or for indications of a slowing down, as conjectured by others.

\section{MODELS}

\section{A. Discrete Nonlinear Schrödinger and Klein-Gordon chains}

In our study we consider various one-dimensional lattice models. The first one is the disordered discrete nonlinear Schrödinger equation (DNLS) described by the Hamiltonian function

$$
\mathcal{H}_{D}=\sum_{l} \epsilon_{l}\left|\psi_{l}\right|^{2}+\frac{\beta}{2}\left|\psi_{l}\right|^{4}-\left(\psi_{l+1} \psi_{l}^{*}+\psi_{l+1}^{*} \psi_{l}\right),
$$

in which $\psi_{l}$ are complex variables, $l$ are the lattice site indices and $\beta \geq 0$ is the nonlinearity strength. The random on-site energies $\epsilon_{l}$ are chosen uniformly from the interval $\left[-\frac{W}{2}, \frac{W}{2}\right]$, with $W$ denoting the disorder strength. The equations of motion are generated by $\dot{\psi}_{l}=\partial \mathcal{H}_{D} / \partial\left(i \psi_{l}^{\star}\right)$ :

$$
i \dot{\psi}_{l}=\epsilon_{l} \psi_{l}+\beta\left|\psi_{l}\right|^{2} \psi_{l}-\psi_{l+1}-\psi_{l-1} .
$$

This above set of equations conserves both the energy of Eq. (1), and the norm $S=\sum_{l}\left|\psi_{l}\right|^{2}$.
The second model we consider is the quartic KleinGordon (KG) lattice, given as

$$
\mathcal{H}_{K}=\sum_{l} \frac{p_{l}^{2}}{2}+\frac{\tilde{\epsilon}_{l}}{2} u_{l}^{2}+\frac{1}{4} u_{l}^{4}+\frac{1}{2 W}\left(u_{l+1}-u_{l}\right)^{2},
$$

where $u_{l}$ and $p_{l}$ respectively are the generalized coordinates and momenta on site $l$, and $\tilde{\epsilon}_{l}$ are chosen uniformly from the interval $\left[\frac{1}{2}, \frac{3}{2}\right]$. The equations of motion are $\ddot{u}_{l}=-\partial \mathcal{H}_{K} / \partial u_{l}$ and yield

$$
\ddot{u}_{l}=-\tilde{\epsilon}_{l} u_{l}-u_{l}^{3}+\frac{1}{W}\left(u_{l+1}+u_{l-1}-2 u_{l}\right) .
$$

This set of equations only conserves the energy of Eq. (3i). The scalar measure of energy resulting from Eq. (3) we shall henceforth label as $H$. This scalar value $H \geq 0$ serves as a control parameter of nonlinearity, similar to $\beta$ for the DNLS case.

For $\beta=0$ and $\psi_{l}=A_{l} \exp (-i \lambda t)$, Eq. (2) reduces to the linear eigenvalue problem

$$
\lambda A_{l}=\epsilon_{l} A_{l}-A_{l-1}-A_{l+1} .
$$

The normalized eigenvectors $A_{\nu, l}\left(\sum_{l} A_{\nu, l}^{2}=1\right)$ are the corresponding normal modes (NMs), and the eigenvalues $\lambda_{\nu}$ are the frequencies of these NMs. The width of the eigenfrequency spectrum $\lambda_{\nu}$ in Eq. (5D) is $\Delta_{D}=W+4$ with $\lambda_{\nu} \in\left[-2-\frac{W}{2}, 2+\frac{W}{2}\right]$. The coefficient $1 /(2 W)$ in Eq. (3) was chosen so that the linear parts of the Hamiltonians, Eqs. (113) would correspond to the same eigenvalue problem. In the limit $H \rightarrow 0$ (in practice by neglecting the nonlinear term $u_{l}^{4} / 4$ ) the KG model of Eq. (3) - with $u_{l}=A_{l} \exp (i \omega t)$ - is reduced to the same linear eigenvalue problem of Eq. (5), under the substitutions $\lambda=W \omega^{2}-W-2$ and $\epsilon_{l}=W\left(\tilde{\epsilon}_{l}-1\right)$. The width of the squared frequency $\omega_{\nu}^{2}$ spectrum is $\Delta_{K}=1+\frac{4}{W}$ with $\omega_{\nu}^{2} \in\left[\frac{1}{2}, \frac{3}{2}+\frac{4}{W}\right]$. Note that $\Delta_{D}=W \Delta_{K}$. As in the case of DNLS, $W$ determines the disorder strength.

The asymptotic spatial decay of an eigenvector is given by $A_{\nu, l} \sim \mathrm{e}^{-l / \xi\left(\lambda_{\nu}\right)}$ where $\xi\left(\lambda_{\nu}\right)$ is the localization length. In the case of weak disorder, $W \rightarrow 0$, the localization length is approximated [17, 21] as $\xi\left(\lambda_{\nu}\right) \leq \xi(0) \approx$ $100 / W^{2}$. The NM participation number $p_{\nu}=1 / \sum_{l} A_{\nu, l}^{4}$ characterizes the spatial extend of the NM. An average measure of this extent is the localization volume $V$, which is of the order of $3.3 \xi(0) \approx 330 / W^{2}$ for weak disorder and unity in the limit of strong disorder, $W \rightarrow \infty$ [17]. The average spacing of eigenvalues of NMs within the range of a localization volume is then $d \approx \Delta / V$, with $\Delta$ being the spectrum width. The two frequency scales $d \leq \Delta$ determine the packet evolution details in the presence of nonlinearity.

In order to write the equations of motion of Hamiltonian (11) in the normal mode space of the system we insert $\psi_{l}=\sum_{\nu} A_{\nu, l} \phi_{\nu}$ in (2), with $\left|\phi_{\nu}\right|^{2}$ denoting the time-dependent amplitude of the $\nu$ th NM. Then, using Eq. (5) and the orthogonality of NMs the equations of 
motion (2) read

$$
i \dot{\phi}_{\nu}=\lambda_{\nu} \phi_{\nu}+\beta \sum_{\nu_{1}, \nu_{2}, \nu_{3}} I_{\nu, \nu_{1}, \nu_{2}, \nu_{3}} \phi_{\nu_{1}}^{*} \phi_{\nu_{2}} \phi_{\nu_{3}}
$$

with the overlap integral

$$
I_{\nu, \nu_{1}, \nu_{2}, \nu_{3}}=\sum_{l} A_{\nu, l} A_{\nu_{1}, l} A_{\nu_{2}, l} A_{\nu_{3}, l} .
$$

The frequency shift of a single site oscillator induced by the nonlinearity is $\delta_{l}=\beta\left|\psi_{l}\right|^{2}$ for the DNLS model. The squared frequency shift of a single site oscillator induced by the nonlinearity for the KG system is $\delta_{l}=\left(3 E_{l}\right) /\left(2 \tilde{\epsilon}_{l}\right)$, with $E_{l}$ being the energy of the oscillator. Since all NMs are exponentially localized in space, each effectively couples to a finite number of neighbor modes. The nonlinear interactions are thus of finite range; however, the strength of this coupling is proportional to the norm (energy) density for the DNLS (KG) model. If the packet spreads far enough, we can generally define two norm (energy) densities: one in real space, $n_{l}=\left|\psi_{l}\right|^{2}\left(E_{l}\right)$ and the other in NM space, $n_{\nu}=\left|\phi_{\nu}\right|^{2}\left(E_{\nu}\right)$. Averaging over realizations, no strong difference is seen between the two, and therefore, we treat them generally as some characteristic norm $(n)$ or energy $(E)$ density. The frequency shift due to nonlinearity is then $\delta_{D} \sim \beta n$ for the DNLS model, while the squared frequency shift is $\delta_{K} \sim 3 E / 2$ for the KG lattice. The basic characteristics of both models are summarized in Table [.

We order the NMs in space by increasing value of the center-of-norm coordinate $X_{\nu}=\sum_{l} l A_{\nu, l}^{2}[9,15,18,19]$. For DNLS we follow normalized norm density distributions $z_{\nu} \equiv\left|\phi_{\nu}\right|^{2} / \sum_{\mu}\left|\phi_{\mu}\right|^{2}$, while for KG we follow normalized energy density distributions $z_{\nu} \equiv E_{\nu} / \sum_{\mu} E_{\mu}$ with $E_{\nu}=\dot{A}_{\nu}^{2} / 2+\omega_{\nu}^{2} A_{\nu}^{2} / 2$, where $A_{\nu}$ is the amplitude of the $\nu$ th NM and $\omega_{\nu}^{2}$ its squared frequency. We measure the second moment $m_{2}=\sum_{\nu}(\nu-\bar{\nu})^{2} z_{\nu}$ (with $\bar{\nu}=\sum_{\nu} \nu z_{\nu}$ ), which quantifies the wave packet's spreading width; the participation number $P=1 / \sum_{\nu} z_{\nu}^{2}$, i.e. the number of the strongest excited modes in $z_{\nu}$; and the compactness index $\zeta=P^{2} / m_{2}$, which quantifies the inhomogeneity of a wave packet. Thermalized distributions have $\zeta \approx 3$, while $\zeta \ll 3$ indicates very inhomogeneous packets, e. g. sparse (with many holes) or partially selftrapped ones (see [15] for more details). In addition, following Anderson's definition of localization [1], we measure the fraction $S_{V}\left(H_{V}\right)$ of the wave packet norm (energy) in a localization volume $V$ around the initially excited state in real space. For a localized state this fraction asymptotically tends to a constant nonzero value, while it goes to zero in the case of delocalization.

\section{B. Fröhlich-Spencer-Wayne chain}

In the limit of strong disorder $(W \rightarrow \infty)$ the DNLS and KG models suffer from increasing computational times needed to observe any nontrivial dynamics. This is because the eigenvectors tend to single site profiles, i.e. the overlap integrals become very small. Fröhlich, Spencer and Wayne (FSW) suggested considering a modified Hamiltonian, which operates directly in normal mode space for the strong disorder limit, but considers artificial rescaled anharmonic interactions between neighboring NMs in order to rescale time 22]:

$$
\mathcal{H}=\sum_{\nu} \frac{p_{\nu}^{2}}{2}+\frac{\epsilon_{\nu}}{2} u_{\nu}^{2}+\frac{1}{4}\left(u_{\nu+1}-u_{\nu}\right)^{4}
$$

where the NMs are equivalent to the single site oscillators. The NM eigenvalues $\epsilon_{\nu}$ are considered to be uncorrelated, also for nearest neighbors. This is different from the DNLS and KG models. Also the FSW chain has only pair interactions between NMs (sites). Note also that the nonlinear part of the FSW Hamiltonian is invariant under any shift $u_{\nu} \rightarrow u_{\nu}+a$, as opposed to the KG model.

\section{Models with spatially inhomogeneous nonlinearity}

We also consider two variants of DNLS and KG models with spatially inhomogeneous nonlinearity. The first type of lattices is composed of linear coupled oscillators except for a central region of length $L$ where nonlinearities are present. We refer to this type as the LNL (LinearNonlinear-Linear) model. The second type is called NLN (Nonlinear-Linear-Nonlinear) and is the exact counterpart of the previous one, since the linear part of the lattice is located at the central $L$ sites.

\section{WAVE PACKET EVOLUTION}

\section{A. Theoretical predictions}

\section{DNLS and $K G$}

The evolution of wave packets in nonlinear disordered chains can be expected to be selftrapped for strong nonlinearities, or show no selftrapping for weaker nonlinearities. The existence of the selftrapping regime was theoretically predicted for the DNLS model in [16] (see also 15 for more details). According to the theorem stated in [16], for large enough nonlinearities $\left(\delta_{D}>\Delta_{D}\right)$ single site excitations cannot uniformly spread over the entire lattice. Consequently, a part of the wave packet will remain localized, although the theorem does not prove that the location of this inhomogeneity is constant in time.

If the nonlinear shift $\delta$ moves the frequencies of some of the initially excited oscillators out of the linear spectrum, it tunes them out of resonance and part of the wave packet will be selftrapped. In our study we consider initial "block" wave packets, where $L$ central oscillators of the lattice are excited having the same norm (energy). 


\begin{tabular}{|c|c|c|c|}
\hline & & DNLS & KG \\
\hline \multicolumn{2}{|l|}{ On-site energies } & $\epsilon_{l} \in\left[-\frac{W}{2}, \frac{W}{2}\right]$ & $\tilde{\epsilon}_{l} \in\left[\frac{1}{2}, \frac{3}{2}\right]$ \\
\hline \multicolumn{2}{|l|}{ Spectrum } & $\lambda_{\nu} \in\left[-2-\frac{W}{2}, 2+\right.$ & $\omega_{\nu}^{2} \in\left[\frac{1}{2}, \frac{3}{2}+\frac{4}{W}\right.$ \\
\hline \multicolumn{2}{|l|}{ Spectrum width $\Delta$} & $\Delta_{D}=W+4$ & $\Delta_{K}=\frac{W+4}{W}$ \\
\hline \multirow{2}{*}{ Localization volume $V\{$} & $W \rightarrow 0$ & \multicolumn{2}{|c|}{$V=\frac{330}{W^{2}}$} \\
\hline & $W \rightarrow \infty$ & \multicolumn{2}{|c|}{$V \sim 1$} \\
\hline \multirow{2}{*}{ Average spacing $d\{$} & \multirow{2}{*}{$\begin{array}{c}W \rightarrow 0 \\
W \rightarrow \infty\end{array}$} & $d_{D} \sim W^{2}$ & $d_{K} \sim W$ \\
\hline & & $d_{D} \sim W$ & $d_{K} \sim$ const. \\
\hline \multicolumn{2}{|c|}{ Nonlinear energy shift $\delta$} & $\delta_{D} \sim \beta n$ & $\delta_{K} \sim \frac{3}{2} E$ \\
\hline
\end{tabular}

TABLE I: Characteristic quantities of the DNLS (1) and the KG (3) models. The dependence on the strength of disorder $W$ of both the localization volume $V$ and of the average spacing $d$ of NMs' eigenvalues within the range of $V$, is given for the limiting cases of weak $W \rightarrow 0$ and strong disorder $W \rightarrow \infty$. Note that $n(E)$ represents a general characteristic norm (energy) of wave packets of the DNLS (KG) model.

Since we consider many random disorder realizations (of the order of few hundreds) we expect that, on average, the linear frequencies of the initially excited lattice sites $\epsilon_{l}$ $\left(\tilde{\epsilon}_{l}\right)$ cover the whole range of permitted values $\left[-\frac{W}{2}, \frac{W}{2}\right]$ $\left(\left[\frac{1}{2}, \frac{3}{2}\right]\right)$. Thus, some of these frequencies are tuned out of resonance if $\delta_{D} \geq 2\left(\delta_{D} \gtrsim 1 / W\right)$. These conditions for the possible appearance of selftrapping are less strict than the theoretically defined ones 15,16$]$ and are, in general, in good agreement with numerical simulations. In particular, the selftrapping regime was numerically observed for single-site excitations [9, 15, 18] and for extended excitations [19], both for the DNLS and the KG models, despite the fact that the KG system conserves only the total energy $E$, and the selftrapping theorem can not be applied there.

When selftrapping is avoided for $\delta_{D}<2\left(\delta_{K} \lesssim 1 / W\right)$, two different spreading regimes were predicted, having different dynamical characteristics: an asymptotic weak chaos regime, and a potential intermediate strong chaos one [20]. Numerical verifications of the existence of these two regimes were presented in [18, 19]. In the weak chaos regime, for $L \geq V$ and $\delta<d$ most of the NMs are weakly interacting with each other. Then the subdiffusive spreading of the wave packet is characterized by $m_{2} \sim t^{1 / 3}$. If the nonlinearity is weak enough to avoid selftrapping, yet strong enough to ensure $\delta>d$, the strong chaos regime is realized. Wave packets in this regime initially spread faster than in the case of weak chaos, with $m_{2} \sim t^{1 / 2}$. Since the norm density drops with further spreading, $\delta$ is dropping in time as well, and eventually the wave packet enters the weak chaos regime, where its evolution is characterized by slower spreading with $m_{2} \sim t^{1 / 3}$. The wave packet evolution in both the weak and the strong chaos spreading regimes is also ex- pected to be characterized by an increase of the participation number as $P \sim t^{\alpha / 2}$ when $m_{2} \sim t^{\alpha}$.

Let as now discuss the spreading of wave packets when $L<V$. The packet will initially spread over the localization volume $V$ during a time interval $\tau_{i n} \sim 2 \pi / d$, even in the absence of nonlinearities [19, 20]. The initial average norm (energy) density $n_{\text {in }}\left(E_{i n}\right)$ of the wave packet is then lowered to $n\left(\tau_{i n}\right) \approx n_{i n} L / V\left(E\left(\tau_{i n}\right) \approx E_{i n} L / V\right)$. The further spreading of the wave packet in the presence of nonlinearities is then determined by these reduced densities. Note that for single-site excitations $(L=1)$, the strong chaos regime therefore completely disappears and the wave packet evolves either in the weak chaos or in the selftrapping regimes $[8,9,15,23]$.

\section{FSW chain}

There are no existing theoretical predictions for wave packet dynamics in the FSW chain. The FSW case can be considered as a strong disorder limit of the KG model, emulating the dynamics of the latter in NM space. However, in the KG and DNLS case, triplet interactions between NMs are present and necessary in order to allow for finite (though small) resonance probabilities for small (but finite) energy and norm densities [17, 20]. Pair interactions will cease to produce NM resonances for sufficiently small densities due to level repulsion within one localization volume [15, 17, 20]. The FSW chain keeps only pair interactions. At the same time, level repulsion between neighboring (interacting) NMs are absent in the FSW case. Therefore, a theoretical analysis similar to the DNLS and KG case 20] appears to be possible. Its details will be considered in a future publication. The 
width of the linear spectrum $\Delta_{F S W}=1$. The average spacing of nearest neighbor eigenvalues $d_{F S W} \sim \Delta_{F S W}$. Therefore, we expect that only the asymptotic regime of weak chaos, and the regime of selftrapping can be expected.

\section{3. $L N L$ and $N L N$ chains}

The LNL chain can be expected to start in a chaotic wave packet spreading regime as long as the wave packet is confined mainly to the finite size $\mathrm{N}$ (nonlinear) part. However, the more the wave packet spreads, the more it extends into the infinitely extended L (linear) parts. Resonances and chaos are therefore confined to the finite $\mathrm{N}$ part. Since distant NMs in the L part are exponentially weakly interacting with the chaotic NMs in the N part, their excitation - if at all - will take times which increase exponentially with growing distance. Therefore, the wave packet will spread (if at all) slower than any power law. Thus, the LNL model is the only model we consider here, where almost trivial slowing down of spreading is expected.

Recently, the dynamics of a similar system was theoretically investigated in 24], where a disordered subsystem of coupled anharmonic oscillators, linearly coupled to an infinite harmonic system, was considered. In this work the conditions which permit the persistence of the discrete breather of the isolated anharmonic system for small but nonvanishing couplings to the harmonic lattice were derived, and cases characterized by energy transfer to the harmonic system were also discussed.

The NLN chain is expected to behave differently. As long as the wave packet is confined mainly to the $\mathrm{L}$ region, the dynamics is regular, and no spreading should occur. For large enough time, some part of the packet will leak out into the $\mathrm{N}$ regions. Therefore, finally spreading of the wave packet should occur.

\section{B. Numerical results}

\section{Methods}

We consider compact DNLS wave packets at $t=0$ spanning a width $L$ centered in the lattice, such that within $L$ there is a constant initial norm of $n_{\text {in }}=1$ and a random phase at each site, while outside the width $L$ the norm density is zero. In the KG case, we excite each site in the width $L$ with the same energy, $E=H / L$, i.e. initial momenta of $p_{l}= \pm \sqrt{2 E}$ with randomly assigned signs.

We use symplectic integration schemes of the SABA family of integrators [15, 25, 26] for the integration of equations (2) and (44). The particular symplectic scheme used for the DNLS model is described in the Appendix. The number of lattice sites $N$ and the integration time step $\tau$ varied between $N=1000$ to $N=2000$ and $\tau=0.01$ to $\tau=0.1$, in order to exclude finite size effects in the wave packet evolution, and in order to reach long integration times up to $10^{7}-10^{9}$ time units with feasible CPU times. In all our simulations, the relative energy and norm errors are kept smaller than $10^{-3}$. For each parameter set we averaged our data over 1000 different disorder realizations, unless otherwise stated, and denote this by $\langle\ldots\rangle$. In particular, we compute $m_{2}$ and $P$, and we smooth $\left\langle\log _{10} m_{2}\right\rangle$ and $\left\langle\log _{10} P\right\rangle$ with a locally weighted regression algorithm [27], and then apply a central finite difference to calculate the local derivatives

$$
\alpha_{m}=\frac{d\left\langle\log _{10} m_{2}\right\rangle}{d \log _{10} t}, \quad \alpha_{P}=\frac{d\left\langle\log _{10} P\right\rangle}{d \log _{10} t} .
$$

\section{Weak disorder}

In this subsection we considerably extend the reports on the observation of weak chaos, strong chaos, the crossover between both, and the selftrapping regime in Ref. 19]. In Fig. 1 we show results for the DNLS model with $W=4$ and $L=V=21$, for six different values of the nonlinearity strength $\beta$. The time evolution of $\left\langle\log _{10} m_{2}(t)\right\rangle$ and $\left\langle\log _{10} P(t)\right\rangle$ is plotted in Figs. 1(a) and $1(\mathrm{~b})$, respectively. The evolution of the compactness index $\langle\zeta(t)\rangle$ is shown in Fig. 1(c). In Figs. 1(d) and 1(e) we plot the time dependence of the numerically computed derivatives $\alpha_{m}(t)$ and $\alpha_{P}(t)$ (9) obtained from the smoothed curves of Figs. 1(a) and 1(b) respectively. Finally, in Fig. 1(f) the values of $\left\langle S_{V}(t)\right\rangle$ are plotted.

The weak chaos dynamics is observed in Fig. 1 for $\beta=0.012$ (black curves) and $\beta=0.04$ (magenta curves). Initially, the wave packets remain localized and all quantities of Fig. 1 are constant with $\alpha_{m}, \alpha_{P}$ being practically zero. After some detrapping time $t_{d}$ the wave packets start to subdiffusively spread with $m_{2} \sim t^{1 / 3}$ and $P \sim t^{1 / 6}$ (Figs. प(a), (b), (d) and (e)). In addition, the compactness index $\langle\zeta\rangle \approx 3$ (Fig. प(c)), indicating that wave packets are well thermalized inside. The tendency towards complete delocalization of wave packets is clearly depicted in the evolution of the averaged norm fraction $\left\langle S_{V}\right\rangle$ which remains at the $L=21$ initially excited sites (Fig. प(f)). After the detrapping time $t_{d}\left\langle S_{V}\right\rangle$ decreases continuously up to the final integration time $t=10^{7}$.

Increasing the value to $\beta=0.18$, the initial spreading dynamics enters the crossover between weak and strong chaos, and a faster spreading is observed. Spreading sets in earlier, the compactness index again indicates thermalized wave packets, and the local derivatives $\alpha_{m}$ and $\alpha_{P}$ increase up to 0.4 and 0.2 respectively, with a possibly very slow decreasing at even larger times. $\left\langle S_{V}\right\rangle$ again continuously decreases to zero indicating complete delocalization.

For $\beta=0.72$, we fully enter the strong chaos regime. Most importantly we observe a saturation of the local exponent $\alpha_{m}$ around the theoretical value $1 / 2$, with a subsequent decay, again as predicted by theory [20], and first observed in [19]. 

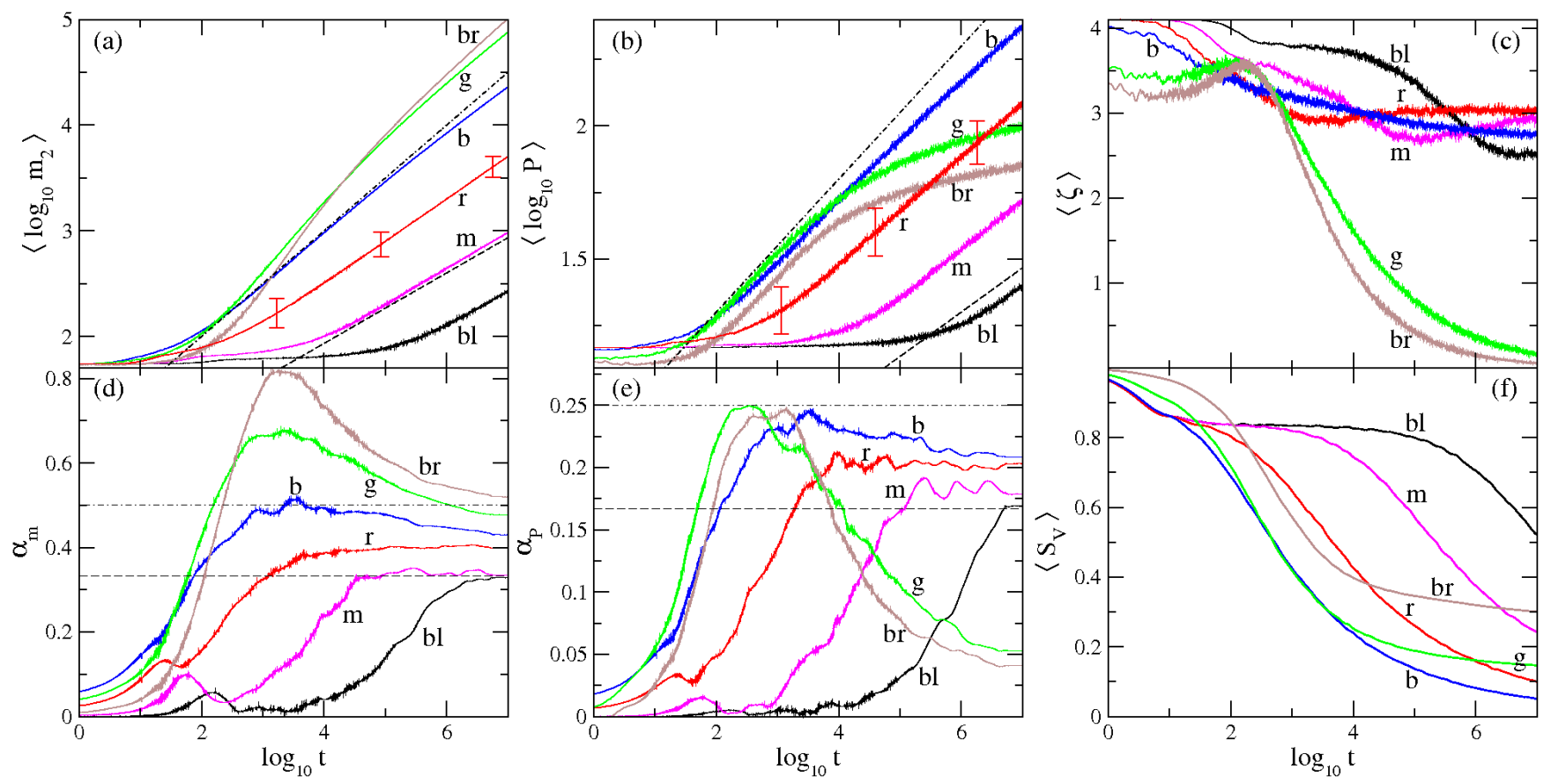

FIG. 1: (Color online) DNLS, $W=4$ : Evolution of (a) $\left\langle\log _{10} m_{2}(t)\right\rangle$, (b) $\left\langle\log _{10} P(t)\right\rangle$, (c) $\langle\zeta(t)\rangle$, (d) finite-difference derivative $\alpha_{m}(t)$ for the smoothed $m_{2}$ data of panel (a), (e) finite-difference derivative $\alpha_{P}(t)$ for the smoothed $P$ data of panel (b), and (f) $\left\langle S_{V}(t)\right\rangle$ for the spreading of wave packets with initial width $L=21$ and $\beta=0.012,0.04,0.18,0.72,3.6,8.4[(\mathrm{bl})$ black; (m) magenta; (r) red; (b) blue; (g) green; (br) brown]. In panels (a), (b), (d) and (e) straight lines correspond to theoretically predicted power laws $m_{2} \sim t^{\alpha}, P \sim t^{\alpha / 2}$ with $\alpha=1 / 3$ (dashed lines) and $\alpha=1 / 2$ (dotted lines). Error bars in panels (a) and (b) denote representative standard deviation errors.

Finally, for $\beta=3.6$ and $\beta=8.4$ (green and brown curves respectively in Fig. 1) the dynamics enters the selftrapping regime. We observe that a part of a wave packet remains localized, while the remainder spreads. The spreading portion results in a continuous increase of $m_{2}$ (Fig. 1(a)) which initially is characterized by large values of $\alpha_{m}>1 / 2$ (Fig. 1(d)). For larger time $\alpha_{m}$ decreases below $1 / 2$. The evolution appears to be rather complex, and is not captured by the theoretical considerations in [20]. The large values of $\alpha_{m}$ may be due to temporal trapping and detrapping processes in this strongly nonequilibrium dynamics of the wave packet, with a final trapped packet fraction remaining. Therefore, we observe that $\left\langle\log _{10} P\right\rangle$ starts to level off (Fig. I(b)), $\alpha_{P}$ tends to very small values (Fig. $1(\mathrm{e}))$ and $\langle\zeta\rangle$ tends to zero (Fig. 1(c)). In Fig. 1(f), we observe that the values of $\left\langle S_{V}\right\rangle$ saturate to higher values for $\beta=8.4$.

For these typical cases of weak chaos $(\beta=0.04)$, strong chaos $(\beta=0.72)$ and selftrapping $(\beta=3.6)$ we present in Figs. 2(a)-(c) the time evolution of the averaged norm density distributions $\left\langle z_{l}\right\rangle$ in real space. All simulations presented in Fig. 2 started from the same initial profile with size $L=V$, therefore the width of the localization volume set by the linear case is the width of the distributions at the shortest times in the plots. In the weak chaos regime (Fig. 2(a)), the wave packets remain close to their initial configuration for some times, as the high $\left\langle z_{l}\right\rangle$ values in the region of the initial excitation indi- cate, followed by delocalization at larger times. Thus, at $t=10^{7}$ the averaged wave packet has spread to about 300 sites with $\left\langle z_{l}\right\rangle>10^{-5}$. Therefore, the wave packet spreads continuously over distances which are an order of magnitude larger than the limits set by the linear theory and destroying Anderson localization. In the case of strong chaos (Fig. 2(b)), spreading is even faster, leading to more extended profiles at $t=10^{7}$ : about 700 sites with $\left\langle z_{l}\right\rangle>10^{-5}$. In the selftrapping regime (Fig. 2(c)), the spreading part of the wave packet covers 1500 sites, with another clearly visible part staying selftrapped at the initial excitation region. The curved fronts in the density plots in Fig. 2 follow from the theoretical prediction $m_{2} \sim t^{\alpha}$ with which leads to a packet width $\mathcal{N} \sim \sqrt{m_{2}}$ and $\mathcal{N} \sim \mathrm{e}^{\left(\alpha \log _{10} t\right) / 2}$.

For the KG model (3) with $W=4$ we present in Fig. 3 similar results to the ones for the DNLS model. For small values of the initial energy density $E=0.01$, the characteristics of the weak chaos regime are observed: $m_{2} \sim t^{1 / 3}$ (black curve in Fig. 3(a)) after a detrapping time $t_{d} \approx 10^{5}$, wave packets remain compact as they spread since $\langle\zeta\rangle \approx 3$ (Fig. [3(b)), and the fraction $\left\langle H_{V}\right\rangle$ of the energy of the initially $L=21$ excited sites decreases (Fig. 3(d)). For $E=0.04$ we enter the crossover region between the weak and the strong chaos regimes, with characteristics similar to the DNLS case. For $E=0.2$ (red curves in Fig. 3) we observe the typical behavior of the strong chaos scenario: spreading is characterized by 


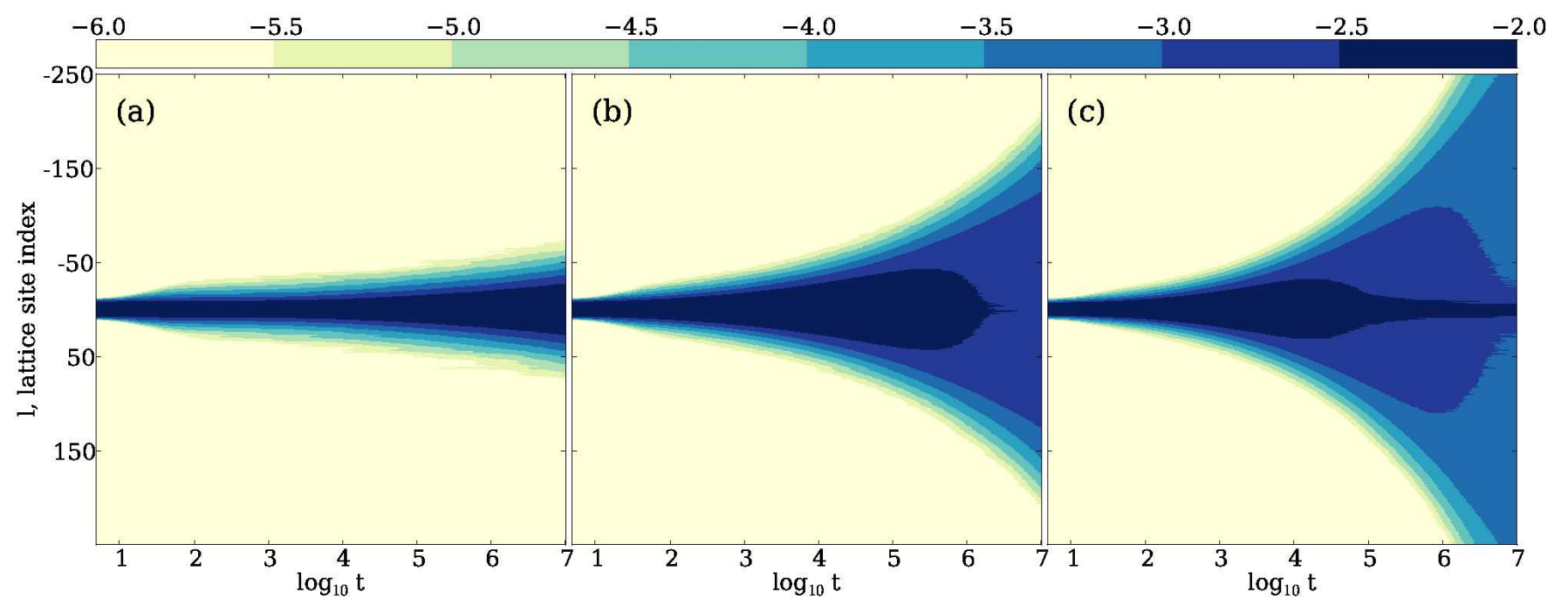

FIG. 2: (Color online) DNLS, $W=4$ : Time evolution of average norm density distributions $\left\langle z_{l}\right\rangle$ in real space for (a) $\beta=0.04$, (b) $\beta=0.72$ and (c) $\beta=3.6$. The color scales shown on top of panels (a)-(c) are used for coloring each lattice site according to its $\log _{10}\left\langle z_{l}\right\rangle$ value.

a saturated $\alpha_{m} \approx 1 / 2$ (Fig. 3(c)) for about two decades $\left(\log _{10} t \approx 3.5-5.5\right)$, followed by a crossover to the weak chaos dynamics with $\alpha_{m}$ decreasing. Getting closer to the selftrapping regime for $E=0.75$ (blue curves in Fig. (3), or being deep inside it for $E=3$ (green curves in Fig. 3) the characteristics of the selftrapping behavior appear, since $\langle\zeta\rangle$ decreases (Fig. 3(b)), and $\left\langle H_{V}\right\rangle$ tends to stabilize to non-zero small values (Fig. [3(d)). Similar to the $\beta=3.6$ and $\beta=8.4$ cases of the DNLS model, the evolution of $m_{2}$ (Fig. B(a)) shows an initial phase of fast growth with $\alpha_{m}>1 / 2$ (Fig. 3(c)) followed by a lowering in the values of $\alpha_{m}$.

Our numerical results are in accord with the predictions of weak and strong chaos regimes, as well as of the crossover from strong to weak chaos. Selftrapping is observed as well, with less understood strongly nonequilibrium dynamics of the trapped and spreading packet parts. We vehemently stress that in all our simulations we never observed any evidence of a wave packet transition from the weak chaos regime, characterized by $\alpha_{m}=1 / 3$, to a subsequent slowing down of spreading, which would lead to $\alpha_{m}<1 / 3$.

\section{Strong disorder}

To search for potential deviations from the predicted spreading laws, we turn to large values of $W$ for the DNLS system. In all our simulations we had $L \geq V$. In particular, we set $L=10$ and considered the cases with $W=15$ and $W=40$. For large values of $W$ we expect only the weak chaos and the selftrapping dynamical regimes to be observed [19]. For each value of $W$ three different values of $\beta$ were considered, one being in the weak chaos regime and the other two in the selftrapping
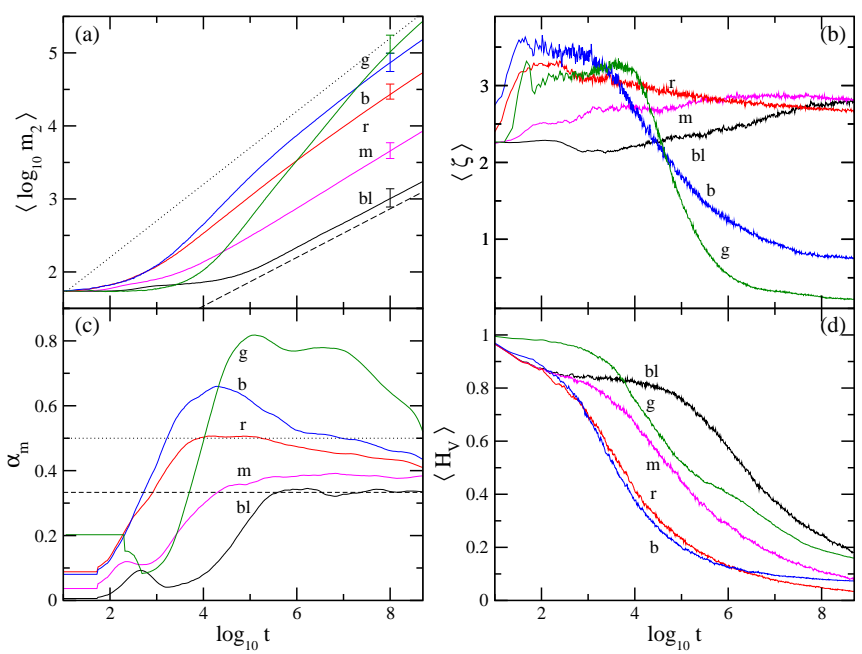

FIG. 3: (Color online) KG, $W=4$ : Evolution of (a) $\left\langle\log _{10} m_{2}(t)\right\rangle$, (b) $\langle\zeta(t)\rangle$, (c) $\alpha_{m}(t)$, and (d) $\left\langle H_{V}(t)\right\rangle$ versus $\log _{10} t$ for the spreading of initially compact wave packets of width $L=21$ with $E=0.01,0.04,0.2,0.75,3$ [(bl) black; (m) magenta; (r) red; (b) blue; (g) green]. In panels (a), and (c) straight lines correspond to the theoretically predicted power laws $m_{2} \sim t^{\alpha}$ with $\alpha=1 / 3$ (dashed lines) and $\alpha=1 / 2$ (dotted lines). Error bars in panel (a) denote representative standard deviation errors.

regime. In particular, we considered $\beta=0.5, \beta=9$ and $\beta=30$ for $W=15$ and $\beta=1, \beta=25$ and $\beta=100$ for $W=40$. The obtained results are shown in Fig. 4 for $W=15$ and in Fig. 5 for $W=40$.

For large $W$, the localization volume $V$ decreases drastically, so that eventually the overlap integrals become small as well. Therefore, the values of $\beta$ at which spreading becomes visible will increase. In the weak chaos 

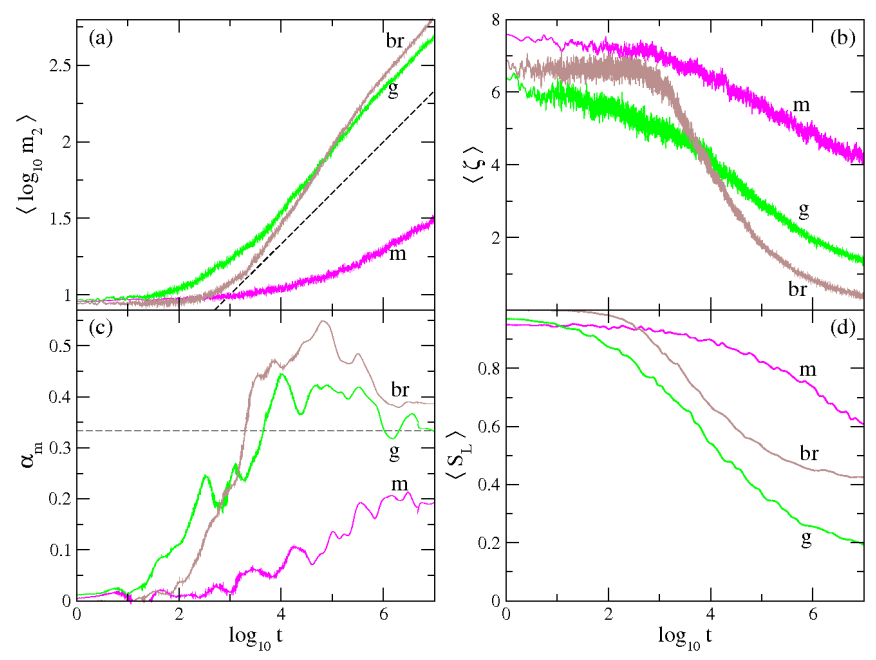

FIG. 4: (Color online) DNLS, $W=15$ : Evolution of (a) $\left\langle\log _{10} m_{2}(t)\right\rangle$, (b) $\langle\zeta(t)\rangle$, (c) $\alpha_{m}(t)$, and (d) $\left\langle S_{L}(t)\right\rangle$ versus $\log _{10} t$ for the spreading of initially compact wave packets of width $L=10$ with $\beta=0.5,9,30[(\mathrm{~m})$ magenta; (g) green; (br) brown]. Mean values are averaged quantities over 100 disorder realizations. In panels (a), and (c) straight lines correspond to theoretically predicted weak chaos behavior $m_{2} \sim t^{1 / 3}$.
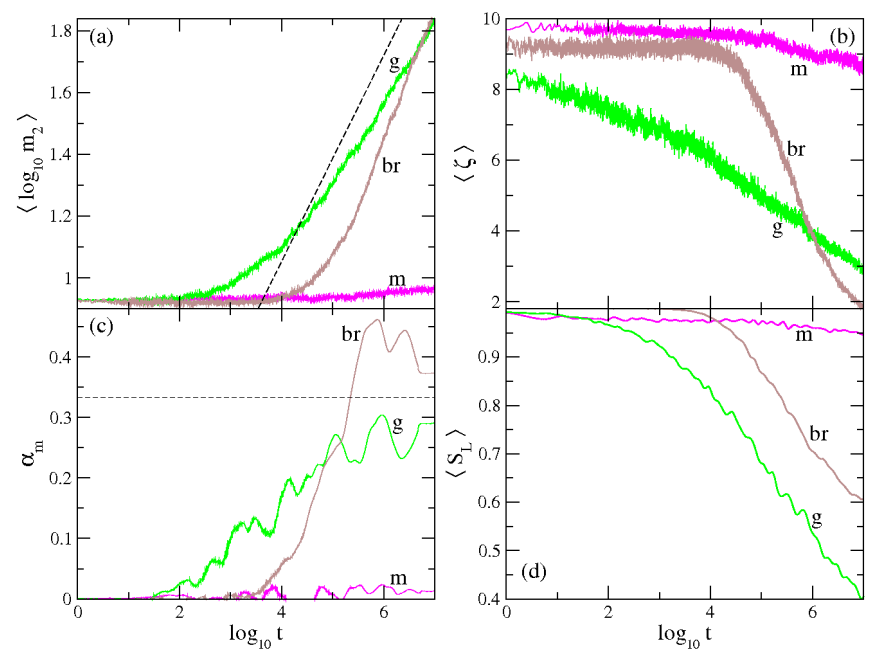

FIG. 5: (Color online) DNLS, $W=40$ : Evolution of (a) $\left\langle\log _{10} m_{2}(t)\right\rangle$, (b) $\langle\zeta(t)\rangle$, (c) $\alpha_{m}(t)$, and (d) $\left\langle S_{L}(t)\right\rangle$ versus $\log _{10} t$ for the spreading of initially compact wave packets of width $L=10$ with $\beta=1,25,100[(\mathrm{~m})$ magenta; (g) green; (br) brown]. Mean values are averaged quantities over 100 disorder realizations. In panels (a), and (c) straight lines correspond to theoretically predicted weak chaos behavior $m_{2} \sim t^{1 / 3}$.

spreading regime the detrapping time $t_{d}$ becomes large and we start observing spreading after long time intervals. For $W=15$ and $\beta=0.5$ we find $t_{d} \approx 10^{3}$ (Fig. 四(a)). The local derivative $\alpha_{m}$ increases from zero showing a tendency to approach the theoretically predicted value $\alpha=1 / 3$ (Fig. 这(c)), and both $\langle\zeta\rangle$ (Fig. $4(\mathrm{~b})$ ) and $\left\langle S_{L}\right\rangle$ (Fig. 4(d)) start to decrease. We note that since $V \sim 1$ for large $W$, we measure the time evolution of the fraction $S_{L}(t)$ of the norm density of the $L=10$ initially excited sites.

The detrapping time $t_{d}$ increases as $W$ increases. This is seen from the results for $W=40, \beta=1$ (magenta curves in Fig. 5). In this case, we have to wait at least up to $t_{d}=10^{5}$ in order to get some evidence that spreading starts, since after that time $\left\langle\log _{10} m_{2}\right\rangle$ starts to slightly grow (Fig. 5 (a)), while $\langle\zeta\rangle$ (Fig. $5(\mathrm{~b})$ ) and $\left\langle S_{L}\right\rangle$ (Fig. [5(d)) start to decrease. This increase of $t_{d}$ happens despite the fact that the nonlinearity strength $\beta$ also increased by a factor of two as compared to the $W=15$ case. Nevertheless even in this case of large $W=40$ we are able to numerically observe the onset of spreading in the weak chaos regime. Increasing $W$ to even higher values pushes $t_{d}$ to values larger than the final integration time $t=10^{7}$ used in our simulations.

With increasing $\beta$ we observe selftrapping, but a part of the wave packet spreads and $m_{2}$ increases (green and

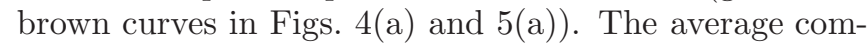
pactness index $\langle\zeta\rangle$ decreases, which is a clear indication that a part of the wave packet remains localized, and reaches smaller final values for larger $\beta$ (Figs. 4(b) and 5(b)). The selftrapping of the wave packets is also clearly

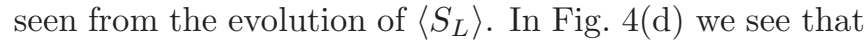
for $W=15, \beta=9$ (green curve) and $\beta=30$ (brown curve) $\left\langle S_{L}\right\rangle$ decreases due to the spreading of a part of wave packets, while, finally it shows a tendency to level off to a positive value, indicating that part of the wave packets remains localized. Similar behaviors of $\left\langle S_{L}\right\rangle$ are observed in Fig. 5 (d) for the $W=40$ case with $\beta=25$ (green curve) and $\beta=100$ (brown curve), although the plateauing of $\left\langle S_{L}\right\rangle$ is not as clear as in Fig. 4 (d). The numerically computed exponents $\alpha_{m}$ exhibit the typical behavior of selftrapping seen in Fig. 1(d). For $W=15$ they increase reaching values larger than $1 / 3$ and afterwards decrease towards $\alpha_{m}=1 / 3$ (Fig. 4(c)). For $W=40$ a similar behavior is observed for $\beta=100$, while for $\beta=25$ $\alpha_{m}$ seems to approach the theoretically predicted value $1 / 3$ from below.

Therefore, even for strong disorder, the dynamics of wave packets evolves according to the theoretical predictions. Most importantly, we do not observe a slowing down of the wave packet below the limits set by the weak chaos regime.

\section{FSW model}

To further probe a possible slowing down of wave packet spreading beyond the limits of weak chaos, we turn to the FSW model (8). In Fig. 6 we present results with initial compact wave packets of width $L=21$, with energy density $E=0.05$, similarly to the KG model (3). We observe that also for this model subdiffusive spreading occurs, because the second moment and the participation number (red and blue curves respectively in Fig. 6(a)) 

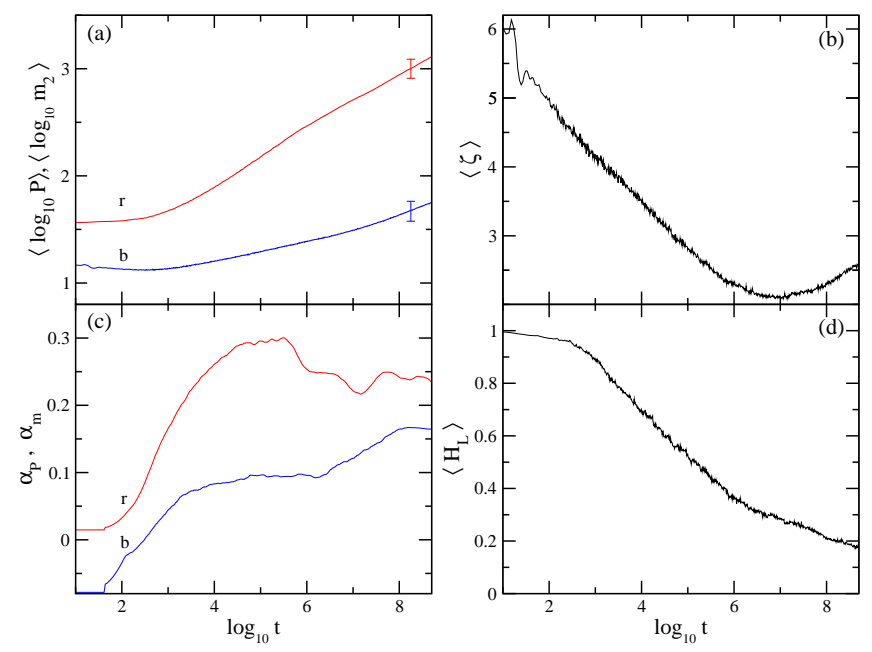

FIG. 6: (Color online) FSW: Evolution of (a) $\left\langle\log _{10} m_{2}(t)\right\rangle$ [(r) red curve] and $\left\langle\log _{10} P(t)\right\rangle[(\mathrm{b})$ blue curve], (b) $\langle\zeta(t)\rangle$, (c) $\alpha_{m}(t)\left[(\mathrm{r})\right.$ red curve] and $\alpha_{P}(t)$ [(b) blue curve], and (f) $\left\langle H_{L}(t)\right\rangle$ versus $\log _{10} t$ for initially compact wave packets of width $L=21$ with $E=0.05$.

increase continuously, and the corresponding exponents $\alpha_{m}$ and $\alpha_{P}$ (Fig. 6(c)) tend to eventual constant nonzero values. The fraction $\left\langle H_{L}\right\rangle$ of energy remaining in the $L=21$ initially excited sites (Fig. 6(d)) decreases as time increases indicating the delocalization of the wave packets. The compactness index (Fig. 6(b)) has a different behavior with respect to what we have seen in the rest of our simulations, as it decreases slowly up to $t \approx 10^{7}$, with a subsequent increase. Therefore, the wave packet is highly inhomogeneous for almost all the integration time, violating the assumptions which are used in the theoretical considerations of weak chaos [20]. The observed subdiffusive spreading may still not be in its final asymptotic range. Still, we again do not see any signature of a slowing down of this subdiffusive process, as was reported in [28] where a similar model was considered. Clearly the FSW model calls for a thorough and independent study.

\section{LNL and NLN models}

In Fig. 7 we present results for the KG, LNL and NLN models for $W=4, L=21$ and $E=0.02$. For comparison we also include the results for the KG model (3) (magenta curves) with $E=0.02$, for which subdiffusive spreading in the weak chaos regime is observed. The KG-NLN model (green curves in Fig. 7) exhibits a similar behavior, since both the second moment (Fig. [7(a)) and the participation number (Fig. $7(b)$ ) start to grow after some detrapping time $t_{d} \approx 10^{5}$. This time is larger than the detrapping time of the KG model $\left(t_{d} \approx 10^{4}\right)$, because a wave packet in the KG-NLN model initially evolves in an almost linear system and only after some large time, when it has spread significantly to the nonlin- ear part of the lattice, spreading takes on characteristics of the purely nonlinear model.

On the other hand the evolution of all quantities of Fig. 7 for the KG-LNL system (red curves) follows the $\mathrm{KG}$ model until $t \approx 10^{4}$, because initially the wave packets evolve in the same nonlinear system. Later on the wave packet enters the L (linear) parts of the system. Thus, spreading starts to retard, and both $\left\langle\log _{10} m_{2}(t)\right\rangle$ (Fig. 7(a)) and $\left\langle\log _{10} P(t)\right\rangle$ (Fig. T(b)) show a characteristic slowing down in the exponents $\alpha_{m}$ (Fig. $7(\mathrm{~d})$ ) and $\alpha_{P}$ (Fig. 7(e)). In addition, $\left\langle S_{V}\right\rangle$ (Fig. 7(f) ) saturates at finite non-zero values, indicating that wave packets tend to localize again. For all three KG models, the values of $\langle\zeta\rangle$ (Fig. $7(\mathrm{c})$ ) show that wave packets do not become sparse and inhomogeneous in the course of time. We obtained similar results for the LNL and NLN DNLS models for $W=4, L=21$ and $\beta=0.04$.

Thus, also for the LNL and NLN models spreading is observed, and only in the case of the LNL models we have observed a slowing down of the spreading, as expected.

\section{SUMMARY AND CONCLUSIONS}

We considered several models of disordered nonlinear one-dimensional lattices and performed extensive numerical simulations of norm (energy) propagations. Since we focused on the dynamical spreading of fronts, we prepared initial block wave packet profiles, having widths equal or larger than the average localization volume defined by the linear problem. While not performed, we expect similar behaviors for initial Gaussian profiles, again where the width (for Gaussians, say the standard deviation) is on par with the average localization volume.

We carefully studied statistical properties of the dynamics, by varying the values of disorder and nonlinearity strengths over a wide interval, and by averaging results over many disorder realizations. Our results agree quite well with our theoretical expectations for the existence of the weak and strong chaos regimes.

The main outcome of our study is that in the presence of nonlinearities we always observe subdiffusive spreading, so that the second moment grows initially as $m_{2} \sim t^{\alpha}$ with $\alpha<1$, showing signs of a crossover to the asymptotic $m_{2} \sim t^{1 / 3}$ law at larger times. Remarkably, subdiffusive spreading is also observed for large disorder strengths, when the localization volume (which defines the number of interacting partner modes) tends to one. Fröhlich-Spencer-Wayne models which take the disorder strength to its infinite limits, are also showing subdiffusive growth. Most remarkably, in none of our studies (except the artificial LNL case) did we encounter a slowing down of spreading beyond the limits set by the weak chaos predictions. Therefore, our numerical data support the conjecture, that the wave packets, once they spread, will do so up to infinite times in a subdiffusive way, bypassing Anderson localization of the linear wave equations. 

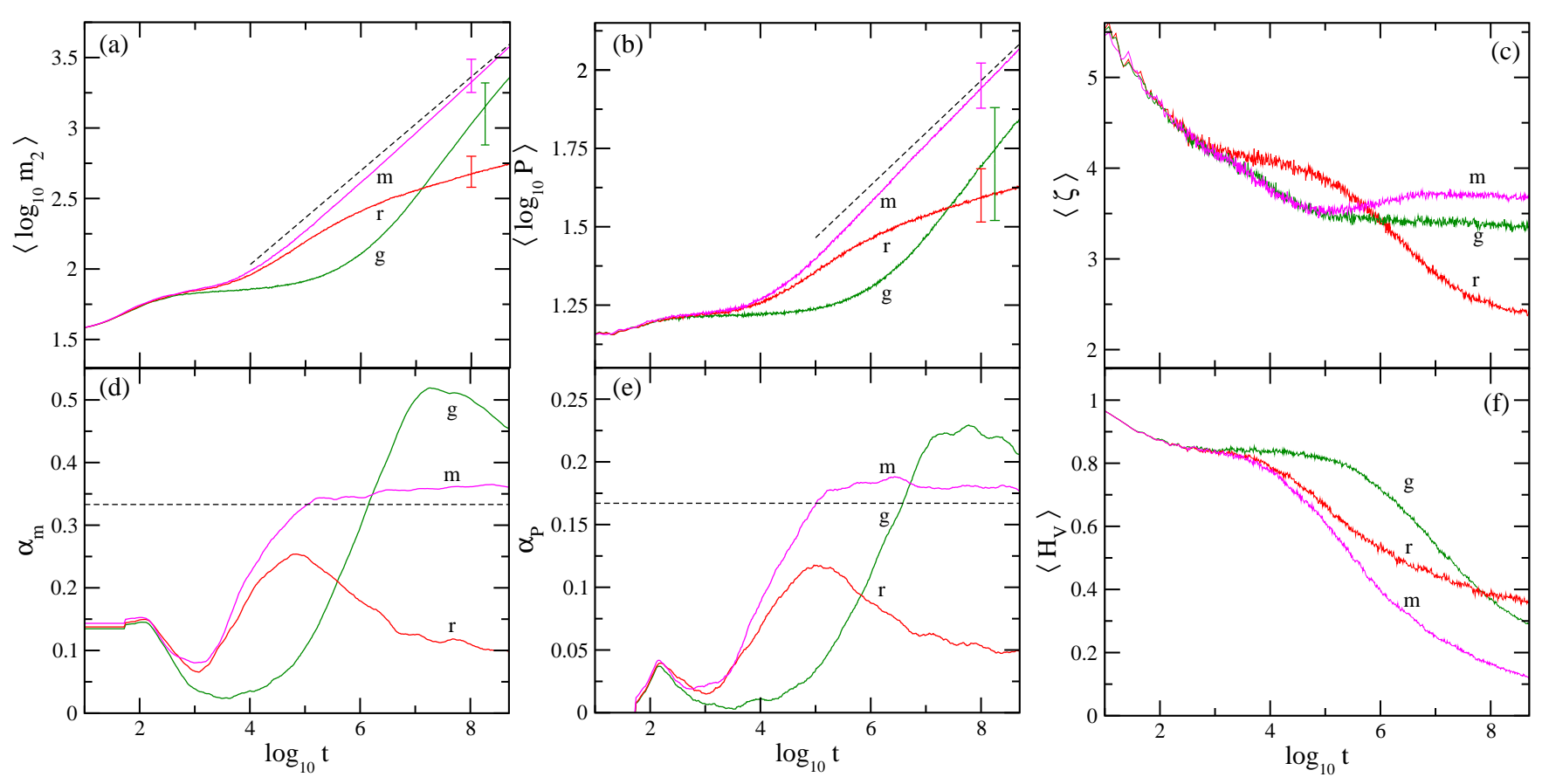

FIG. 7: (Color online) Evolution of (a) $\left\langle\log _{10} m_{2}(t)\right\rangle$, (b) $\left\langle\log _{10} P(t)\right\rangle$, (c) $\langle\zeta(t)\rangle$, (d) $\alpha_{m}(t)$, (e) $\alpha_{P}(t)$, and (f) $\left\langle H_{V}(t)\right\rangle$ versus $\log _{10} t$ for the spreading of initially compact wave packets of width $L=21$ with $W=4$ and $E=0.02$ in the KG $[(\mathrm{m})$ magenta curves], the KG-NLN [(g) green curves] and the KG-LNL [(r) red curves] models. In panels (a), (b), (d) and (e) straight lines correspond to theoretically predicted power laws $m_{2} \sim t^{1 / 3}, P \sim t^{1 / 6}$ of the weak chaos regime. Error bars in panels (a) and (b) denote representative one standard deviation errors.

The only cases where spreading shows a tendency to stop are the LNL models, for which nonlinearities are absent everywhere except inside a finite-size central region, where the initial wave packet is launched. In these models, when wave packets have spread substantially, their chaotic component in the central region of the lattice becomes weak, and distant normal modes in the linear parts of the system are exponentially weakly coupled to the central nonlinear region.

When the nonlinearity strength tends to smaller values, waiting (detrapping) times for wave packet spreading of compact initial excitations increase beyond the detection capabilities of our computational tools. The corresponding question of whether a KAM regime can be entered at finite nonlinearity strength was addressed in [12] and is analyzed in detail in a forthcoming work [29].

\section{Acknowledgments}

We thank S. Aubry, M. Mulansky, A. Pikovsky, R. Schilling and D. Shepelyansky for useful discussions. Ch. S. was partly supported by the European research project "Complex Matter", funded by the GSRT of the Ministry Education of Greece under the ERA-Network Complexity Program.

\section{Appendix A: Symplectic integration of the DNLS equations}

We first discuss a novel PQ method which we designed to integrate the DNLS equations locally. Previously used methods employ a transformation of the wave function from real into Fourier space and back, at each integration step. These transformations induce small but observable corrections in the tails of the wave packet, which slowly but steadily grow in time. In such a case we will have to stop the integration once this noisy background reaches a substantial level. The PQ method avoids the generation of this background by simply not performing the Fourier transformation. Instead the PQ method integrates the DNLS equations in real space.

The canonical transformation

$$
\psi_{l}=\frac{1}{\sqrt{2}}\left(q_{l}+i p_{l}\right)
$$

of the complex variable $\psi_{l}$ in Eq. (1) transforms (1) into

$$
\mathcal{H}_{D}=\sum_{l} \frac{\epsilon_{l}}{2}\left(q_{l}^{2}+p_{l}^{2}\right)+\frac{\beta}{8}\left(q_{l}^{2}+p_{l}^{2}\right)^{2}-\left(q_{l+1} q_{l}+p_{l+1} p_{l}\right) .
$$

where $q_{l}$ and $p_{l}$ are generalized coordinates and momenta, respectively.

If a Hamiltonian function can be split into two integrable parts, then a symplectic integration scheme can 
be used for the integration of its equations of motion. One possible splitting of the DNLS Hamiltonian (A2) into two separate Hamiltonian functions $A$ and $B$ is

$$
\begin{aligned}
A & =\sum_{l} \frac{\epsilon_{l}}{2}\left(q_{l}^{2}+p_{l}^{2}\right)+\frac{\beta}{8}\left(q_{l}^{2}+p_{l}^{2}\right)^{2} \\
B & =-\sum_{l}\left(q_{l+1} q_{l}+p_{l+1} p_{l}\right) .
\end{aligned}
$$

Hamiltonian $A$ is integrable and the operator $e^{\tau L_{A}}$ which propagates the set of initial conditions $\left(q_{l}, p_{l}\right)$ at time $t$, to their final values $\left(q_{l}^{\prime}, p_{l}^{\prime}\right)$ at time $t+\tau$ is

$$
e^{\tau L_{A}}:\left\{\begin{array}{l}
q_{l}^{\prime}=q_{l} \cos \left(\alpha_{l} \tau\right)+p_{l} \sin \left(\alpha_{l} \tau\right) \\
p_{l}^{\prime}=p_{l} \cos \left(\alpha_{l} \tau\right)-q_{l} \sin \left(\alpha_{l} \tau\right)
\end{array},\right.
$$

with $\alpha_{l}=\epsilon_{l}+\beta\left(q_{l}^{2}+p_{l}^{2}\right) / 2$. Hamiltonian $B$ of Eq. A3 is not integrable, thus the operator $e^{\tau L_{B}}$ cannot be written explicitly. If we consider $B$ as a separate Hamiltonian function and again split it as $B=P+Q$, the component parts

$$
P=-\sum_{l} p_{l+1} p_{l}, Q=-\sum_{l} q_{l+1} q_{l} .
$$

are integrable, under the corresponding operators

$$
e^{\tau L_{P}}:\left\{\begin{array}{l}
p_{l}^{\prime}=p_{l} \\
q_{l}^{\prime}=q_{l}-\left(p_{l-1}+p_{l+1}\right) \tau
\end{array}\right.
$$

and

$$
e^{\tau L_{Q}}:\left\{\begin{array}{l}
q_{l}^{\prime}=q_{l} \\
p_{l}^{\prime}=p_{l}+\left(q_{l-1}+q_{l+1}\right) \tau
\end{array}\right.
$$

This technique of splitting the Hamiltonian into multiple parts has been used in different applications of symplectic integrators (see for example [30]).

In our simulations we successively apply the $\mathrm{SBAB}_{2}$ symplectic integrator [15, 25, 26] twice: first for the split $\left(\mathcal{H}_{D}=A+B\right)$ of the DNLS Hamiltonian, Eq. (A2), and second for the split $B=P+Q$ in Eq. (A3). The solution for the equations of motion from the Hamiltonian Eq. A2 is thus approximated by the application of 13 simple operators on an initial condition $\left(q_{l}, p_{l}\right)$, since

$$
\begin{aligned}
e^{\tau \mathcal{H}_{D}} & =e^{\tau(A+B)} \approx e^{d_{1} \tau L_{A}} e^{c_{2} \tau L_{B}} e^{d_{2} \tau L_{A}} e^{c_{2} \tau L_{B}} e^{d_{1} \tau L_{A}} \approx \\
& \approx e^{d_{1} \tau L_{A}} e^{d_{1} c_{2} \tau L_{P}} e^{c_{2}^{2} \tau L_{Q}} e^{c_{2} d_{2} \tau L_{P}} e^{c_{2}^{2} \tau L_{Q}} e^{d_{1} c_{2} \tau L_{P}} e^{d_{2} \tau L_{A}} e^{d_{1} c_{2} \tau L_{P}} e^{c_{2}^{2} \tau L_{Q}} e^{c_{2} d_{2} \tau L_{P}} e^{c_{2}^{2} \tau L_{Q}} e^{d_{1} c_{2} \tau L_{P}} e^{d_{1} \tau L_{A}}
\end{aligned}
$$

with the $\mathrm{SBAB}_{2}$ coefficients [25] of $d_{1}=1 / 6, d_{2}=2 / 3 \quad$ and $c_{2}=1 / 2$.

[1] P. W. Anderson, Phys. Rev. 109, 1492 (1958).

[2] J. Billy, V. Josse, Z. Zuo, A. Bernard, B. Hambrecht, P. Lugan, D. Clément, L. Sanchez-Palencia, P. Bouyer, A. Aspect, Nature 453, 891 (2008).

[3] G. Roati, C. D'Errico, L. Fallani, M. Fattori, C. Fort, M. Zaccanti, G. Modugno, M. Modugno, M. Inguscio, Nature 453, 895 (2008).

[4] T. Schwartz, G. Bartal, S. Fishman, M. Segev, Nature 446, 52 (2007).

[5] Y. Lahini, A. Avidan, F. Pozzi, M. Sorel, R. Morandotti, D.N. Christodoulides, Y. Silberberg, Phys. Rev. Lett. 100, 013906 (2008).

[6] R. Dalichaouch, J.P. Armstrong, S. Schultz, P.M. Platzman, S.L. Mccall, Nature 354, 53 (1991); C. Dembowski, H.-D. Gräf, R. Hofferbert, H. Rehfeld, A. Richter, T. Weiland, Phys. Rev. E 60, 3942 (1999); J.D. Bodyfelt, M. C. Zheng, T. Kottos, U. Kuhl, H.-J. Stöckmann, Phys. Rev. Lett. 102, 253901 (2009).

[7] M. Molina, Phys. Rev. B 58, 12547 (1998).

[8] A. S. Pikovsky and D. L. Shepelyansky, Phys. Rev. Lett. 100, 094101 (2008).

[9] S. Flach, D. O. Krimer and Ch. Skokos, Phys. Rev. Lett. 102, 024101 (2009).
[10] D. M. Basko, Ann. Phys., (2011), doi:10.1016/j.aop.2011.02.004.

[11] I. Březinová, L. A. Collins, K. Ludwig, B. I. Schneider and J. Burgdörfer, Phys. Rev. A 83, 043611 (2011).

[12] M. Johansson, G. Kopidakis and S. Aubry, Europhys. Lett. 91, 50001 (2010).

[13] A. Pikovsky and S. Fishman, Phys. Rev. E 83, 025201(R) (2011).

[14] S. Fishman, Y. Krivolapov, A. Soffer, Nonlinearity 22, 2861 (2009).

[15] Ch. Skokos, D. O. Krimer, S. Komineas and S. Flach, Phys. Rev. E 79, 056211 (2009).

[16] G. Kopidakis, S. Komineas, S. Flach and S. Aubry, Phys. Rev. Lett. 100, 084103 (2008).

[17] D. O. Krimer and S. Flach, Phys. Rev. E 82, 046221 (2010).

[18] Ch. Skokos and S. Flach, Phys. Rev. E 82, 016208 (2010).

[19] T. V. Laptyeva, J. D. Bodyfelt, D. O. Krimer, Ch. Skokos and S. Flach, Europhys. Lett. 91, 30001 (2010).

[20] S. Flach, Chem. Phys. 375, 548 (2010).

[21] B. Kramer and A. MacKinnon, Rep. Prog. Phys. 56, 1469 (1993).

[22] J. Fröhlich, T. Spencer, and C.E. Wayne, J. Stat. Phys. 
42, $247(1986)$

[23] H. Veksler, Y. Krivolapov and S. Fishman, Phys. Rev. E 80, 037201 (2009).

[24] S. Aubry and R. Schilling, Physica D 283, 2045 (2009).

[25] J. Laskar and P. Robutel, Cel. Mech. Dyn. Astr. 80, 39 (2001).

[26] Ch. Skokos and E. Gerlach, Phys. Rev. E 82, 036704 (2010).

[27] W. S. Cleveland and S. J. Devlin, J. Am. Stat. Assoc.
83, 596 (1988).

[28] M. Mulansky, K. Ahnert and A. Pikovsky, Phys. Rev. E 83, 026205 (2011).

[29] M. V. Ivanchenko, T. V. Laptyeva and S. Flach, in preparation (2011).

[30] K. Goździewski, S. Breiter and W. Borczyk, Mon. Not. R. Astron. Soc. 383, 989 (2008). 